\title{
On Variational Formulations with Rigid-body Constraints for Finite Elasticity: Applications to 2D and 3D Finite Element Simulations
}

\author{
Heng Chi, Glaucio H. Paulino \\ School of Civil and Environmental Engineering, Georgia Institute of Technology, 790 Atlantic Drive, Atlanta, GA, 30332, USA
}

\begin{abstract}
We investigate a recently proposed variational principle with rigid-body constraints and present an extension of its implementation in three dimensional finite elasticity problems. Through numerical examples, we illustrate that the proposed variational principle with rigid-body constraints is applicable to both single field and mixed finite elements of arbitrary order and geometry, e.g. triangular/tetrahedral and quadrilateral/hexagonal elements, in two and three dimensions. Moreover, we demonstrate that, as compared to the commonly adopted approach of discretizing the rigid domains with standard finite elements, the proposed formulation requires neither discretization nor numerical integration in the interior of each rigid domain. As a comparative result, the variational formulation may reduce the total number of degrees of freedom of the resulting finite element system and provide improved accuracy.
\end{abstract}

Keywords: variational principles, finite elastostatics, rigid inclusions, 3D FEA

\section{Introduction}

The presence of stiff fillers is prevalent in engineering and biological materials. Although of different nature, those stiff fillers typically have significantly stiffer mechanical responses, in the order of five orders of magnitudes or possibly more, than the underlying soft matrix. From a computational modeling perspective, a commonly adopted approach in the literature to model the stiffer fillers is to discretize them with standard finite elements, e.g., triangular and quadrilateral elements, and assign them much larger moduli (typically a thousand times or more) than those of the soft matrix $[1,2,3,4]$. However, this approach adds unnecessary degrees of freedom (DOFs) (since the fillers are much stiffer and do not experience any strain) to the resulting system of equations and thus inevitably increases the computational expense. Alternatively, recent research suggests that, making use of the polygonal discretization, each filler can be treated as one single polygonal finite element (the "ngon" approach) [5]. However, as demonstrated in [6], since the fillers discretized with one polygonal elements tend to be "mechanically softer" than the standard elements, numerical integration of higher order is required in conjunction with larger moduli, which increases the number of integration points and degrade the conditioning of the finite element (FE) stiffness matrix. On the other hand, it is worthwhile mentioning that with the ideas recently proposed in the Virtual Element Method (VEM) $[7,8,9,10,11]$, the integration issue associated with the above mentioned "n-gon" approach could be possibly avoided, as demonstrated in [11].

More recently, a different methodology has been proposed to model the stiff fillers [6]. Instead of being approximated as a material phase of high moduli, the stiff fillers are effectively idealized as rigid bodies. Accordingly, a variational formulation is put forward, which takes into account the presence of rigid spatial domains in elastic solids by describing each of them as a set of kinematic constraints over the boundary. Therefore, no discretization is needed in the interior of each rigid domain. While the set of constraints only consists of kinematic quantities (displacement field), other key information, such as the average stress field and boundary trac- 
tions, can be extracted from the Lagrange multipliers related to the constraints [6]. In reference [6], the FE implementation aspects and numerical examples of the variational principle have been presented in two dimensions (2D) and for lower order polygonal elements. In this work, we provide a complete extension of the FE implementation of the variational principle to three dimensions (3D), and demonstrate its application to both standard and higher order finite elements.

The remainder of the paper is organized as follows: Section 2 reviews the variational principle proposed in [6] with both displacement-based and two-field mixed formulations. Section 3 presents the extension of the FE implementation of the variational principle to $3 \mathrm{D}$. Section 4 demonstrates the applications of the variational principles in studying physically-based examples with standard finite elements of various orders, including: $(i)$ the nonlinear elastic response of a filled elastomer reinforced with a random isotropic distribution of spherical particles, and (ii) the onset of cavitation in a fiber reinforced elastomer. Section 5 provides some concluding remarks. Finally, we provide two appendices which complement the paper.

Before proceeding with the technical sections, we briefly and partially introduce the notation adopted in this paper. For any given variable (or field) $g$, be it a tensor of any order, we denote by $\delta g$ its variation. For any function (or functional) $f(g)$ that depends on $g$, we write $\mathrm{D} f(g) \cdot \delta g$ to denote its derivative (or directional derivative) with respect to $g$. Moreover, for any subset $E$ of a given spatial domain $\Omega, E \subset \Omega$, we denote by $|E|$ its area or volume, and by $\partial E$ its boundary. We define $\langle\cdot\rangle_{E}$ as the average operator:

$$
\langle\cdot\rangle_{E} \doteq \frac{1}{|E|} \int_{E}(\cdot) \mathrm{d} \mathbf{X}
$$

If the average is taken over the whole domain $\Omega$, we use instead the notation $\langle\cdot\rangle$ with the subscript $\Omega$ omitted. We shall also use $|\cdot|$ to denote the standard determinant operator for a matrix.

\section{Variational formulations with rigid-body con- straints: Finite elastostatics}

Consider an elastic solid in its undeformed and stressfree configuration that occupies a domain $\Omega$ with bound- ary $\partial \Omega$. A displacement field $\mathbf{u}^{0}$ is prescribed on part of the boundary $\partial \Omega^{\mathbf{X}}$, whereas a surface traction $\mathbf{t}$ (per unit undeformed surface) is prescribed on the complementary part of the boundary $\partial \Omega^{\sigma}$. Furthermore, we assume that the elastic solid contains $N$ constitutively rigid regions $\Omega_{1}^{(2)}, \ldots, \Omega_{N}^{(2)}$, that are disconnected and collectively occupy the domain $\Omega^{(2)}=\Omega_{1}^{(2)} \cup \ldots \cup \Omega_{N}^{(2)}$. The remaining non-rigid part of the solid is denoted by $\Omega^{(1)}$ and is taken to be characterized by a hyperelastic energy $W^{(1)}$, which is a non-negative, quasi-convex function of the deformation gradient F. Accordingly, the first Piola-Kirchhoff stress tensor at each material point in $\Omega^{(1)}$ is given by the constitutive relation:

$$
\mathbf{P}(\mathbf{X})=\frac{\partial W^{(1)}}{\partial \mathbf{F}}(\mathbf{X}, \mathbf{F})
$$

The interface of material discontinuity between the rigid domain $\Omega_{i}^{(2)}$ and the non-rigid part $\Omega^{(1)}$ is denoted as $\Gamma^{i}$, so that $\Gamma^{1} \cap \ldots \cap \Gamma^{N}=\varnothing$. A schematic description of the solid is provided in Fig. 1. Throughout this paper, we mainly focus on the case where the presence of body forces is neglected, however, the generalization of the variational formulation incorporating presence of body forces can be found in the Appendix A. Throughout this paper, a Lagrangian description of the fields is adopted.

Exploiting the fact that the motion of the rigid domains can be completely described by the motion on their boundaries, the basic ideology of the variational formulations with rigid-body constraints is to constrain the motions on the boundary $\Gamma^{i}$ of each rigid domain to be a rigid-body motion, and consider the standard variational formulation in the non-rigid domain $\Omega^{(1)}$. In particular, the variational principle considers a restricted set of kinematically admissible displacements $\mathcal{K}^{R}$ :

$$
\begin{array}{r}
\mathcal{K}^{R}=\left\{\mathbf{u}: \text { on } \partial \Omega^{\mathbf{X}}, \mathbf{u}=\mathbf{u}^{0}, \text { and on } \Gamma^{i}, i=1, \ldots, N,\right. \\
\mathbf{u}=\mathbf{u}_{0}^{i}+\mathbf{H}^{i} \mathbf{X}, \mathbf{H}^{i T} \mathbf{H}^{i}+\mathbf{H}^{i}+\mathbf{H}^{i T}=0 \\
\text { where } \left.\mathbf{u}_{0}^{i} \in \mathbb{R}^{d}, \mathbf{H}^{i} \in \mathbb{R}^{d \times d}\right\}
\end{array}
$$

where the constant vector $\mathbf{u}_{0}^{i} \in \mathbb{R}^{d}$ and constant secondorder tensor $\mathbf{H}^{i} \in \mathbb{R}^{d \times d}$ characterize the rigid-body translation and rotation of each rigid domain $\Omega_{i}^{(2)}$, respectively. In this paper, this concept is applied in the context 
of both standard displacement-based and mixed formulations.

\subsection{The displacement-based formulation with rigid-body constraints}

The so-called displacement-based formulation of finite elastostatics considers the displacement field $\mathbf{u}$ as the only independent field; the deformation gradient is accordingly written as $\mathbf{F}(\mathbf{u})=\mathbf{I}+\nabla \mathbf{u}$, where $\nabla$ denotes the gradient operator with respect to the undeformed configuration and $\mathbf{I}$ stands for the identity in the space of second-order tensors. When incorporating the presence of rigid domains, the classical principle of minimum potential energy becomes seeking the equilibrium displacement field $\widehat{\mathbf{u}}$ such that,

$$
\Pi^{(1)}(\widehat{\mathbf{u}})=\min _{\mathbf{u} \in \mathcal{K}^{R}} \Pi^{(1)}(\mathbf{u})
$$

where

$$
\Pi^{(1)}(\mathbf{u})=\int_{\Omega^{(1)}} W^{(1)}(\mathbf{X}, \mathbf{F}(\mathbf{u})) \mathrm{d} \mathbf{X}-\int_{\partial \Omega^{\sigma}} \mathbf{t} \cdot \mathbf{u} \mathrm{d} S
$$

is the potential energy in the non-rigid domain $\Omega^{(1)}$.

By introducing two sets of Lagrange multipliers, $\lambda^{i}$ and $\boldsymbol{\Sigma}^{i}, i=1, \ldots, N$, which are assumed to be continuous vectors and constant symmetric second-order tensors, respectively, we recast the above constrained optimization as:

$$
\begin{array}{r}
\min _{\mathbf{u} \in \mathcal{K}\left(\Omega^{(1)}\right)} \min _{\mathbf{u}_{0}^{i} \in \mathbb{R}^{d}} \min _{\mathbf{H}^{i} \in \mathbb{R}^{d \times d}} \operatorname{stat}_{\boldsymbol{\lambda}^{i} \in\left[C^{0}\left(\Gamma^{i}\right)\right]^{d}} \operatorname{stat}_{\boldsymbol{\Sigma}^{i} \in \mathbb{R}_{\text {symm }}^{d \times d}} \\
\mathcal{L}\left(\mathbf{u}, \mathbf{u}_{0}^{i}, \mathbf{H}^{i}, \boldsymbol{\lambda}^{i}, \boldsymbol{\Sigma}^{i}\right)
\end{array}
$$

with

$$
\begin{aligned}
\mathcal{L}\left(\mathbf{u}, \mathbf{u}_{0}^{i}, \mathbf{H}^{i}, \boldsymbol{\lambda}^{i}, \boldsymbol{\Sigma}^{i}\right) & =\Pi^{(1)}(\mathbf{u}) \\
+\sum_{i=1}^{N} \frac{1}{2}\left|\Omega_{i}^{(2)}\right| \boldsymbol{\Sigma}^{i}:\left(\mathbf{H}^{i T} \mathbf{H}^{i}+\mathbf{H}^{i}+\mathbf{H}^{i T}\right) & \\
& +\sum_{i=1}^{N} \int_{\Gamma^{i}} \boldsymbol{\lambda}^{i} \cdot\left(\mathbf{u}-\mathbf{u}_{0}^{i}-\mathbf{H}^{i} \mathbf{X}\right) \mathrm{d} S,
\end{aligned}
$$

where $\mathcal{K}\left(\Omega^{(1)}\right)$ is the space of kinematically admissible displacement in $\Omega^{(1)}$ and stat stands for stationarity with respected to its argument.

It is worthwhile noting that, by looking into the strong form of the Euler-Lagrange equations of (6), the physical meaning of the two sets of Lagrange multipliers are identified [6]. More specifically, $\boldsymbol{\lambda}^{i}$ is found as the traction field on the interface $\Gamma^{i}$, and $\Sigma^{i}$ corresponds to the average first Piola-Kirchhoff stress over the rigid domain $\Omega_{i}^{(2)}$ through

$$
\left(\mathbf{H}^{i}+\mathbf{I}\right) \boldsymbol{\Sigma}^{i}=\langle\mathbf{P}(\mathbf{X})\rangle_{\Omega_{i}^{(2)}} \quad i=1, \ldots, N
$$

We emphasize that although we only consider the kinematic constraints on the boundary of each rigid domain $\Omega_{i}^{(2)}$ in the variational formulation, key information in the interior of each rigid domain $\Omega_{i}^{(2)}$ can be directly extracted from the Lagrange multiplier fields, which is essential for many problems, such as computational homogenization. Furthermore, we remark that with the application of the method of Lagrange multipliers, the nature of the solution we seek is altered from pure minimum to a combination of minimum and stationary fields, as shown in (6), which makes it unsuitable for stability analysis because we on longer have positive definite stiffness matrices for stability any more. As an alternative approach, the penalty method can be adopted for the constraint optimization problem (4), which treats the constraints in (4) with penalty terms instead of the Lagrange multipliers. With the penalty method [12], although the constraints (i.e. rigid-body motion of each rigid domain) are no longer strictly satisfied, standard approaches for stability analysis can be directly employed, which searches for the loss of positive definiteness of the stiffness matrix. For better performance, one may adopt a combination of both the method of Lagrange multipliers and the penalty method when stability analysis is of interest, in which the constraints $\mathbf{u}=\mathbf{u}_{0}^{i}+\mathbf{H}^{i} \mathbf{X}$ in (3) are treated with the Lagrange multipliers whereas the remaining ones are considered with penalization terms. Notice that the constraints $\mathbf{u}=\mathbf{u}_{0}^{i}+\mathbf{H}^{i} \mathbf{X}$ can be practically rewritten as linear constraints, and therefore can be condensed out in the FE implementation (see Section 3) without altering the minimum nature of the solution.

The weak form of the Euler-Lagrange equations of (6) can be found in [6], and therefore is not listed here for the sake of simplicity. 
2.2. The two-field mixed formulation with rigid-body constraints

Following the same concept and procedure as the displacement-based formulation, the two-field mixed formulation (presented here in the context of the $\overline{\mathbf{F}}$ formulation $[13,14,15,16,17,5]$; while the $\mathbf{F}$-formulation can be found in [5]) for elastostatics incorporating rigid domains reads as:

$\min _{\mathbf{u} \in \mathcal{K}\left(\Omega^{(1)}\right)} \min _{\mathbf{u}_{0}^{i} \in \mathbb{R}^{d}} \min _{\mathbf{H}^{i} \in \mathbb{R}^{d \times d}} \operatorname{stat}_{\boldsymbol{\lambda}^{i} \in\left[C^{0}\left(\Gamma^{i}\right)\right]^{d}} \operatorname{stat}_{\boldsymbol{\Sigma}^{i} \in \mathbb{R}_{\text {symm }}^{d \times d}} \max _{p \in \mathcal{Q}\left(\Omega^{(1)}\right)}$

$$
\overline{\mathcal{L}}\left(\mathbf{u}, \mathbf{u}_{0}^{i}, \mathbf{H}^{i}, \boldsymbol{\lambda}^{i}, \boldsymbol{\Sigma}^{i}, p\right)
$$

with

$$
\begin{aligned}
& \overline{\mathcal{L}}\left(\mathbf{u}, \mathbf{u}_{0}^{i}, \mathbf{H}^{i}, \boldsymbol{\lambda}^{i}, \boldsymbol{\Sigma}^{i}, p\right)=\bar{\Pi}^{(1)}(\mathbf{u}, p) \\
&+\sum_{i=1}^{N} \frac{1}{2}\left|\Omega_{i}^{(2)}\right| \boldsymbol{\Sigma}^{i}:\left(\mathbf{H}^{i T} \mathbf{H}^{i}+\mathbf{H}^{i}+\mathbf{H}^{i T}\right) \\
&+\sum_{i=1}^{N} \int_{\Gamma^{i}} \boldsymbol{\lambda}^{i} \cdot\left(\mathbf{u}-\mathbf{u}_{0}^{i}-\mathbf{H}^{i} \mathbf{X}\right) \mathrm{d} S,
\end{aligned}
$$

and

$$
\begin{aligned}
\bar{\Pi}^{(1)}(\mathbf{u}, p) & =\int_{\Omega^{(1)}}\left\{-\bar{W}^{(1) *}(\mathbf{X}, \overline{\mathbf{F}}(\mathbf{u}), p)\right. \\
& +p[\operatorname{det} \mathbf{F}(\mathbf{u})-1]\} \mathrm{d} \mathbf{X}-\int_{\partial \Omega^{\sigma}} \mathbf{t} \cdot \mathbf{u} \mathrm{d} S .
\end{aligned}
$$

The space $\mathcal{Q}\left(\Omega^{(1)}\right)$ consists of square integrable function in $\Omega^{(1)}$ and $p$ is an additional pressure field introduced via the partial Legendre transforms

$$
\bar{W}^{*}(\mathbf{X}, \overline{\mathbf{F}}, p)=\max _{J}\{p(J-1)-\bar{W}(\mathbf{X}, \overline{\mathbf{F}}, J)\},
$$

where the function $\bar{W}(\mathbf{X}, \overline{\mathbf{F}}, J)$ is such that $W(\mathbf{X}, \mathbf{F})=$ $\bar{W}(\mathbf{X}, \overline{\mathbf{F}}, J)$ when $J=\operatorname{det} \mathbf{F}$.

Again, the Lagrange multiplier field $\lambda^{i}$ is the traction field on the interface $\Gamma^{i}$, whereas $\boldsymbol{\Sigma}^{i}$ relates the volume average of the first Piola-Kirchhoff stress over $\Omega_{i}^{(2)}$ via the relation (8). The weak form of the Euler-Lagrange equations of the above variational problem can also be found in [6] and thus is not repeated here.

\section{Finite element implementation}

Along the lines of the discussion in [5] for the FE approximation and implementation for the rigid-body constraint formulation in $2 \mathrm{D}$, this section presents a complete extension of the FE implementation of the rigidbody constraint formulation to $3 \mathrm{D}$.
Consider a decomposition $\Omega_{h}$ of the domain $\Omega$. In particular, the discretization of the non-rigid domain $\Omega_{h}^{(1)}$ contains non-overlapping finite elements of arbitrary geometry (triangular/tetrahedral, quadrilateral/hexagonal and polygonal/polyhedral elements) and order, and each discrete rigid domain $\Omega_{i h}^{(2)}$ is considered as a general polygon/polyhedral with its boundary $\Gamma_{h}^{i}$.

It is worthwhile stating the discrete spaces for the unknown field before we proceed to the FE implementation aspects. First, the global displacement space $\mathcal{K}_{h, k}$ in the non-rigid domain has the following definition

$$
\begin{aligned}
\mathcal{K}_{h, k}=\left\{\mathbf{u}_{h} \in\left[C^{0}\left(\Omega_{h}^{(1)}\right)\right]^{3} \cap \mathcal{K}\left(\Omega^{(1)}\right):\right. & \\
& \left.\left.\mathbf{u}_{h}\right|_{E} \in\left[\mathcal{V}_{k}(E)\right]^{3}, \forall E \in \Omega_{h}^{(1)}\right\}
\end{aligned}
$$

where $\mathcal{V}_{k}(E)$ is the local FE space over element $E$. For a local displacement space of order $k$, it is $k$ th order complete, i.e.

$$
\mathcal{P}_{k}(E) \subseteq \mathcal{V}_{k}(E)
$$

where $\mathcal{P}_{k}(E)$ are polynomial space of order $k$, and its functions possess $k$ th-order variation on the edges of $E$. Example of $\mathcal{V}_{k}(E)$ are the conforming $\mathcal{P}_{k}(E)$ space on triangle/tetrahedral elements, the space constructed by iso-parametric mapping on quadrilateral/hexagonal elements, and the barycentric coordinates on polygonal/polyhedral elements.

Second, for the mixed formulation, various choices of the discrete pressure can be adopted together with the rigid-body constraint formulation $[18,19]$. In this paper, the discrete pressure field $p_{h}$ is approximated as

$$
\begin{array}{r}
\mathcal{Q}_{h, k-1}=\left\{q_{h} \in \mathcal{L}^{2}\left(\Omega_{h}^{(1)}\right):\left.q_{h}\right|_{E} \in \mathcal{V}_{k-1}(E),\right. \\
\left.\forall E \in \Omega_{h}^{(1)}\right\} .
\end{array}
$$

which resembles the Taylor-Hood element in fluid problems. [18, 20]. For higher order cases, we observe that the above approximation implies that the discrete pressure field (of order $k-1$ ) is continuous across the element boundaries. For the linear case, we define $\mathcal{V}_{0}(E)=\mathcal{P}_{0}$ by convention, meaning that the discrete pressure field is constant over each element but discontinuous across the element boundaries. As a result, the elements coincide with the standard lower order mixed finite elements. 
Additionally, the discrete space for the Lagrange multiplier $\lambda_{h}^{i}$ is defined as a piecewise space on $\Gamma_{h}^{i}, \mathcal{W}_{k}\left(\Gamma_{h}^{i}\right)$, such that

$$
\mathcal{W}_{k}\left(\Gamma_{h}^{i}\right)=\left\{\boldsymbol{\lambda}_{h}^{i} \in\left[C^{0}\left(\Gamma_{h}^{i}\right)\right]^{d}:\left.\boldsymbol{\lambda}_{h}^{i}\right|_{F} \in\left[\mathcal{V}_{k}^{F}\right]^{3}\right\}
$$

where $F$ denotes the faces of $\Gamma_{h}^{i}$, respectively, and $\mathcal{V}_{k}^{F}$ is a local space defined on face $F$. Notice that the above definition implies that $\boldsymbol{\lambda}_{h}^{i}$ possess $k$ th-order polynomial variations on the edges of each $\Gamma_{h}^{i}$.

In the discussion that follows, the FE approximation and implementation of the rigid-body constraints given by Eqs. (6) and (9) mainly deal with those terms that are associated with rigid domains. The other terms for the non-rigid domain, on the other hand, follow the standard FE procedures. In this section, we will mainly focus on the two terms associated with each rigid domain into the following two terms, as well of their relevant variations:

$$
\begin{aligned}
& \int_{\Gamma_{h}^{i}} \boldsymbol{\lambda}_{h}^{i} \cdot\left(\mathbf{u}_{h}-\mathbf{u}_{0}^{i}-\mathbf{H}^{i} \mathbf{X}\right) \mathrm{d} S, \\
& \frac{1}{2}\left|\Omega_{i h}^{(2)}\right|\left(\mathbf{H}^{i T} \mathbf{H}^{i}+\mathbf{H}^{i}+\mathbf{H}^{i T}\right): \boldsymbol{\Sigma}^{i}
\end{aligned}
$$

In order to reduce the number of unknown variables, we condense $\mathbf{u}_{0}^{i}$ and $\mathbf{H}^{i}$ out of the above terms, and therefore out of the FE system, by introducing a set of "reference nodes", $\widetilde{\mathbf{X}}_{r}^{i}$ on $\Gamma_{h}^{i}$ for each rigid domain $\Omega_{i h}^{(2)}$. Each set consists of three (or four) in 2D (or 3D ) "reference nodes", which are required to be non-collinear (or non-coplanar). A schematic description of the "reference nodes" can be found in Figs. 2 (a) and (b) in 2D and 3D, respectively.

In $3 \mathrm{D}$ case, with a given set of "reference nodes", any linear field $g(\mathbf{X})$ can be interpolated as

$$
g(\mathbf{X})=\sum_{r=1}^{4} \phi_{r}^{i}(\mathbf{X}) \widetilde{g}_{, r}^{i}
$$

where $\widetilde{g}_{, r}^{i}=g\left(\widetilde{\mathbf{X}}_{r}^{i}\right)$ is introduced to denote the degrees of freedom of $g$ on the "reference nodes".

Following the same definition of those in 2D [6], the interpolants $\phi_{r}^{i}(\mathbf{X})$ in 3D are defined as:

$\phi_{1}^{i}(\mathbf{X})=\frac{\left|\begin{array}{cccc}1 & x & y & z \\ 1 & \widetilde{x}_{2}^{i} & \widetilde{y}_{2}^{i} & \widetilde{z}_{2}^{i} \\ 1 & \widetilde{x}_{3}^{i} & \widetilde{y}_{3}^{i} & \widetilde{z}_{3}^{i} \\ 1 & \widetilde{x}_{4}^{i} & \widetilde{y}_{4}^{i} & \widetilde{z}_{4}^{i}\end{array}\right|}{\widetilde{\phi}^{i}}, \phi_{2}^{i}(\mathbf{X})=\frac{\left|\begin{array}{cccc}1 & \widetilde{x}_{1}^{i} & \widetilde{y}_{1}^{i} & \widetilde{z}_{1}^{i} \\ 1 & x & y & z \\ 1 & \widetilde{x}_{3}^{i} & \widetilde{y}_{3}^{i} & \widetilde{z}_{3}^{i} \\ 1 & \widetilde{x}_{4}^{i} & \widetilde{y}_{4}^{i} & \widetilde{z}_{4}^{i}\end{array}\right|}{\widetilde{\phi}^{i}}$,
$\phi_{3}^{i}(\mathbf{X})=\frac{\left|\begin{array}{cccc}1 & \widetilde{x}_{1}^{i} & \widetilde{y}_{1}^{i} & \widetilde{z}_{1}^{i} \\ 1 & \widetilde{x}_{2}^{i} & \widetilde{y}_{2}^{i} & \widetilde{z}_{2}^{i} \\ 1 & x & y & z \\ 1 & \widetilde{x}_{4}^{i} & \widetilde{y}_{4}^{i} & \widetilde{z}_{4}^{i}\end{array}\right|}{\widetilde{\phi}^{i}}, \phi_{4}^{i}(\mathbf{X})=\frac{\left|\begin{array}{cccc}1 & \widetilde{x}_{1}^{i} & \widetilde{y}_{1}^{i} & \widetilde{z}_{1}^{i} \\ 1 & \widetilde{x}_{2}^{i} & \widetilde{y}_{2}^{i} & \widetilde{z}_{2}^{i} \\ 1 & \widetilde{x}_{3}^{i} & \widetilde{y}_{3}^{i} & \widetilde{z}_{3}^{i} \\ 1 & x & y & z\end{array}\right|}{\widetilde{\phi}^{i}}$,

with

$$
\widetilde{\phi}^{i}=\left|\begin{array}{cccc}
1 & \widetilde{x}_{1}^{i} & \widetilde{y}_{1}^{i} & \widetilde{z}_{1}^{i} \\
1 & \widetilde{x}_{2}^{i} & \widetilde{y}_{2}^{i} & \widetilde{z}_{2}^{i} \\
1 & \widetilde{x}_{3}^{i} & \widetilde{y}_{3}^{i} & \widetilde{z}_{3}^{i} \\
1 & \widetilde{x}_{4}^{i} & \widetilde{y}_{4}^{i} & \widetilde{z}_{4}^{i}
\end{array}\right|,
$$

and $\mathbf{X}=\{x, y, z\}^{T}$.

With the help of the "reference nodes" and their associated interpolants, we are able to rewrite Eq. (17) into the following matrix representation:

$$
\int_{\Gamma_{h}^{i}} \boldsymbol{\lambda}_{h}^{i} \cdot\left(\mathbf{u}_{h}-\mathbf{u}_{0}^{i}-\mathbf{H}^{i} \mathbf{X}\right) \mathrm{d} S=\boldsymbol{\Lambda}^{i T} \mathbf{C}^{i} \mathbf{U}^{i}
$$

where arrays $\boldsymbol{\Lambda}^{i} \in \mathbb{R}^{3 n-12}$ and $\mathbf{U}^{i} \in \mathbb{R}^{3 n}$ are of the form

$$
\boldsymbol{\Lambda}^{i}=\left\{\begin{array}{c}
\Lambda_{h x, 1}^{i} \\
\Lambda_{h y, 1}^{i} \\
\Lambda_{h z, 1}^{i} \\
\vdots \\
\Lambda_{h x, n-4}^{i} \\
\Lambda_{h y, n-4}^{i} \\
\Lambda_{h z, n-4}^{i}
\end{array}\right\} \text { and } \mathbf{U}^{i}=\left\{\begin{array}{c}
u_{h x, 1}^{i} \\
u_{h y, 1}^{i} \\
u_{h z, 1}^{i} \\
\vdots \\
\widetilde{u}_{h x, 1}^{i} \\
\widetilde{u}_{h y, 1} \\
\widetilde{u}_{h z, 1} \\
\vdots
\end{array}\right\}
$$

and $\Lambda_{h x, j}^{i}, \Lambda_{h y, j}^{i}, \Lambda_{h z, j}^{i}$ are the components of nodal traction associated with vertex $\mathbf{X}_{j}^{i}$. We remark that the nodal traction $\Lambda_{h x, j}^{i}, \Lambda_{h y, j}^{i}, \Lambda_{h z, j}^{i}$ has similar nature to the nodal force for surface traction in the standard FE literature $[19,21,22,23,24]$ - it is directly solved in the FE system. We also remark that with the "reference nodes" technique, nodal tractions associated with the "reference nodes" are excluded from the vector $\boldsymbol{\Lambda}^{i}$, and therefore are not solved the in the FE system. If the nodal tractions of all the nodes in the rigid domain $\Omega_{i}^{(2)}$ are of interest, for instance, in interfacial debonding problems, one needs to describe the rigid-body motion $\mathbf{u}_{0}^{i}+\mathbf{H}^{i} \mathbf{X}$ directly (by considering the components of the vector and tensor as unknown variables), instead of representing them with the displacements on the "reference nodes". Furthermore, if the surface traction field $\boldsymbol{\lambda}_{h}^{i}$ is of 
interest, it may be reconstructed from the nodal tractions

as a postprocessing step. The coefficient matrix $\mathbf{C}^{i}$ is a

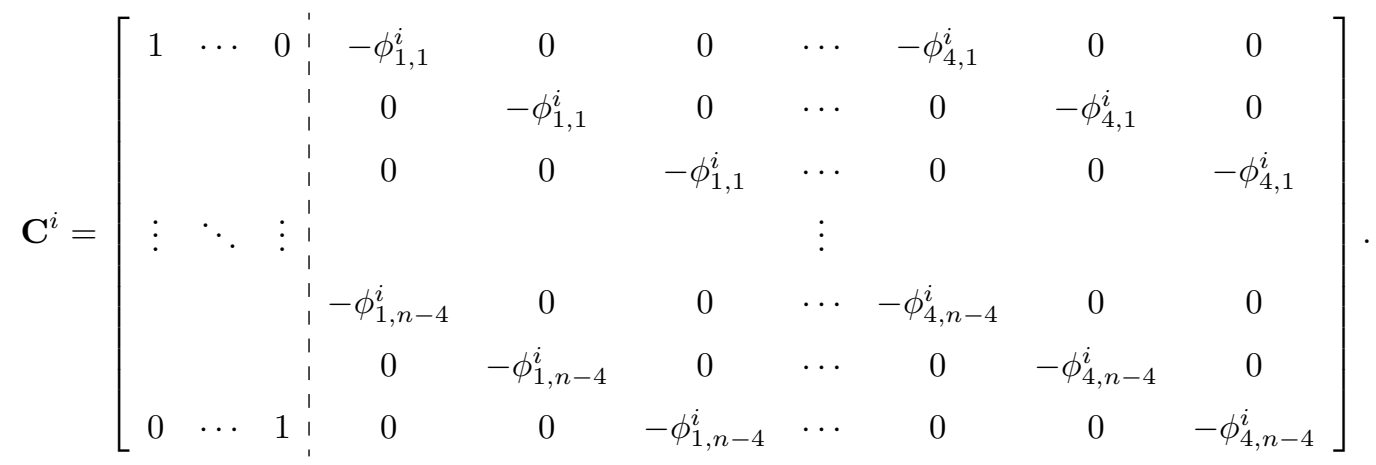

The displacement gradient $\mathbf{H}^{i}$ in each rigid domain can be expressed in matrix notations as well:

$\mathbf{H}^{i}=\left[\begin{array}{ccc}\widetilde{u}_{x, 1}^{i} & & \widetilde{u}_{x, 4}^{i} \\ \widetilde{u}_{y, 1}^{i} & \cdots & \widetilde{u}_{y, 4}^{i} \\ \widetilde{u}_{z, 1}^{i} & & \widetilde{u}_{z, 4}^{i}\end{array}\right]\left[\begin{array}{ccc}\partial \phi_{1}^{i} / \partial x & \partial \phi_{1}^{i} / \partial y & \partial \phi_{1}^{i} / \partial z \\ \vdots & \\ \partial \phi_{4}^{i} / \partial x & \partial \phi_{4}^{i} / \partial y & \partial \phi_{4}^{i} / \partial z\end{array}\right]$

Plugging in the above simplified terms (their variations can be obtained easily in a consistent manner) into the weak forms of displacement-based, we recast the Galerkin FE approximation in the form:

$$
\begin{aligned}
\mathrm{D} \mathcal{L}_{h} \cdot \delta \mathbf{u}_{h} & =\oint_{\Omega_{h}^{(1)}} \frac{\partial W^{(1)}}{\partial \mathbf{F}}\left(\mathbf{X}, \mathbf{F}\left(\mathbf{u}_{h}\right)\right): \nabla\left(\delta \mathbf{u}_{h}\right) \mathrm{d} \mathbf{X} \\
-\oint_{\partial \Omega_{h}^{\sigma}} \mathbf{t} & \cdot \delta \mathbf{u}_{h} \mathrm{~d} S+\sum_{i=1}^{N}\left|\Omega_{i h}^{(2)}\right|\left(\widetilde{\mathbf{U}}^{i} \mathbf{B}^{i}\right) \boldsymbol{\Sigma}^{i}:\left(\widetilde{\left.\delta \mathbf{U}^{i} \mathbf{B}^{i}\right)}\right. \\
& +\sum_{i=1}^{N} \delta \mathbf{U}^{i T} \mathbf{C}^{i T} \boldsymbol{\Lambda}^{i}=0, \quad \forall \delta \mathbf{u}_{h} \in \mathcal{K}_{h, k}^{0},
\end{aligned}
$$

$\mathrm{D} \mathcal{L}_{h} \cdot \delta \boldsymbol{\Sigma}^{i}=\frac{1}{2}\left|\Omega_{i h}^{(2)}\right|\left(\mathbf{B}^{i T} \widetilde{\mathbf{U}}^{i T} \widetilde{\mathbf{U}}^{i} \mathbf{B}^{i}+\widetilde{\mathbf{U}}^{i} \mathbf{B}^{i}+\mathbf{B}^{i T} \widetilde{\mathbf{U}}^{i T}\right)$

$$
: \delta \boldsymbol{\Sigma}^{i}=0, \quad \forall \delta \boldsymbol{\Sigma}^{i} \in \mathbb{R}_{\mathrm{symm}}^{3 \times 3}
$$

$\mathrm{D} \mathcal{L}_{h} \cdot \delta \boldsymbol{\Lambda}^{i}=\delta \boldsymbol{\Lambda}^{i T} \mathbf{C}^{i} \mathbf{U}^{i}=0, \quad \forall \delta \boldsymbol{\Lambda}^{i} \in \mathbb{R}^{3 n-12}$.

Similarly, the mixed formulation takes the form:

$$
\begin{gathered}
\mathrm{D} \overline{\mathcal{L}}_{h} \cdot \delta \mathbf{u}_{h}=\oint_{\Omega_{h}^{(1)}}\left[\frac{-\partial \bar{W}^{*(1)}}{\partial \mathbf{F}}\left(\mathbf{X}, \overline{\mathbf{F}}\left(\mathbf{u}_{h}\right), p_{h}\right)\right. \\
\left.+p_{h} \frac{\partial}{\partial \mathbf{F}}\left(\operatorname{det} \mathbf{F}\left(\mathbf{u}_{h}\right)\right)\right]: \nabla\left(\delta \mathbf{u}_{h}\right) \mathrm{d} \mathbf{X}-\oint_{\partial \Omega_{h}^{\sigma}} \mathbf{t} \cdot \delta \mathbf{u}_{h} \mathrm{~d} S \\
+\sum_{i=1}^{N}\left|\Omega_{i h}^{(2)}\right|\left(\widetilde{\mathbf{U}}^{i} \mathbf{B}^{i}\right) \boldsymbol{\Sigma}^{i}:\left(\widetilde{\delta \mathbf{U}}^{i} \mathbf{B}^{i}\right)+\sum_{i=1}^{N} \delta \mathbf{U}^{i T} \mathbf{C}^{i T} \boldsymbol{\Lambda}^{i}=0, \\
\forall \delta \mathbf{u}_{h} \in \mathcal{K}_{h, k}^{0}\left(\Omega^{(1)}\right),
\end{gathered}
$$

$$
\mathrm{D} \overline{\mathcal{L}}_{h} \cdot \delta p_{h}=\oint_{\Omega_{h}^{(1)}}\left[\operatorname{det} \mathbf{F}\left(\mathbf{u}_{h}\right)-1\right.
$$

$$
\begin{array}{r}
\left.-\frac{\partial \bar{W}^{*(1)}}{\partial p}\left(\mathbf{X}, \mathbf{F}\left(\mathbf{u}_{h}\right), p_{h}\right)\right] \delta p_{h}=0, \\
\forall \delta p_{h} \in \mathcal{Q}_{h, k-1}\left(\Omega^{(1)}\right),
\end{array}
$$

$$
\begin{array}{r}
\mathrm{D} \overline{\mathcal{L}}_{h} \cdot \delta \boldsymbol{\Sigma}^{i}=\frac{1}{2}\left|\Omega_{i h}^{(2)}\right|\left(\mathbf{B}^{i T} \widetilde{\mathbf{U}}^{i T} \widetilde{\mathbf{U}}^{i} \mathbf{B}^{i}+\widetilde{\mathbf{U}}^{i} \mathbf{B}^{i}+\mathbf{B}^{i T} \widetilde{\mathbf{U}}^{i T}\right) \\
: \delta \boldsymbol{\Sigma}^{i}=0, \quad \forall \delta \boldsymbol{\Sigma}^{i} \in \mathbb{R}_{\text {symm }}^{3 \times 3},
\end{array}
$$

$\mathrm{D} \overline{\mathcal{L}}_{h} \cdot \delta \boldsymbol{\lambda}^{i}=\delta \boldsymbol{\Lambda}^{i T} \mathbf{C}^{i} \mathbf{U}^{i}, \quad \forall \delta \boldsymbol{\Lambda}^{i} \in \mathbb{R}^{3 n-12}$.

The above Galerkin approximations (26)-(28) and (29)(32) can be readily used in FE implementations. We note that while terms related to each rigid domain reduce to a set of algebraic constraining equations, we take the standard FE procedure for the non-rigid domain $\Omega_{h}^{(1)}$. In the above equations, we have made use of the notation

$$
\oint_{\Omega_{h}^{(1)}}=\sum_{E \in \Omega_{h}^{(1)}} \oint_{E}
$$

to indicate standard numerical evaluation of the integrals over $\Omega_{h}^{(1)}$, which account for elemental contributions following the standard assembly rules (see, e.g., $[19,21,22,23])$. The symbol $\oint$ is employed to indicate the numerical evaluation of integrals over the part of boundary $\partial \Omega_{h}^{\sigma}$ where the traction boundary condition is applied.

\section{Numerical examples}

In the sequel, we present two applications of the rigidbody constraint formulation in the study of physical behavior of filled elastomers: $(i)$ a qualitative study regarding the onset of cavitation in a fiber reinforced elastomer, and $(i i)$ the nonlinear elastic response of a compressible 
elastomer reinforced with a random isotropic distribution of spherical rigid particles. The first application uses a mixed formulation and the second one a displacementbased formulation. These applications aim to demonstrate the ability of the rigid-body constraint formulation to be used in conjunction with finite elements of arbitrary geometry and order in both $2 \mathrm{D}$ and 3D. Throughout, the compressible neo-Hookean material model is adopted, whose stored-energy function has the form:

$$
W(\mathbf{F})=\frac{\mu}{2}[\overline{\mathbf{F}}: \overline{\mathbf{F}}-3]+\frac{\kappa}{2}(\operatorname{det} \mathbf{F}-1)^{2},
$$

where $\mu$ and $\kappa$ are the initial shear and bulk moduli, respectively. The standard Newtwon-Raphson algorithm is used to solve the nonlinear system of equations and each loading step is regarded as convergent once the norm of the residual reduces below $10^{-6}$ times that of the initial residual.

\subsection{Onset of cavitation in fiber reinforced elastomers}

One interesting phenomenon of the filled elastomers is cavitation, that is, the sudden growth of pre-existing defects into large cavities under sufficiently large loads [25]. In their experiment, Cho and Gent [26] investigated this phenomenon in fiber reinforced elastomers by some ingenious experimental setups. They made specimens in which two steel tubes of length $L$ and diameter $D$ were parallelly aligned at an initial distance $d$ and bonded by a layer of transparent silicone rubber, as depicted in Fig. 3 (a). They then subject the specimen to uniaxial tension transverse to the tubes and observed the onset of cavitation in the region in between the two tubes through the transparent rubber. As an example, Fig. 3 (b) shows a photograph of the cavity formed between the two steel tubes in the specimen with an initial distance of $d=0.43$ mm.

This example adopts the experiment setup of Cho and Gent [26] and idealize it to a 2D plane-strain model. The $2 \mathrm{D}$ model is shown in Fig. 3 (c) and (d) in undeformed and deformed configurations. Because the main purpose of example is to demonstrate the effectiveness and robustness of the rigid body constraint formulation, we only chose one set of experiments among the several sets of experiments that were reported, which has the following dimensions: $L=12.5 \mathrm{~mm}, D=9.5 \mathrm{~mm}$ and $d=0.43 \mathrm{~mm}$. The size of the block $H$ is chosen to be $H=300 \mathrm{~mm}$, which is found to be large enough to avoid the interaction between the fibers an the outer boundary. The macroscopic strain for such problem is defined as $\bar{\varepsilon} \doteq h / H-1$, where $h$ is the deformed length of the block in the direction of applied tension. To allow for cavitation, three vacuous defects that are circular in shape with an initial radius $R_{c}=2 \mu \mathrm{m}$ are introduced at three critical locations in the numerical model, according to previous works on the onset of cavitation in such problems [27]. Two defects are placed $1 \mu \mathrm{m}$ away from the inner sides of the fibers/rubber interfaces and the other at the midpoint in between the fibers (steel tubes).

The shear modulus $\mu$ of the silicone rubber is taken to be 1 and its bulk modulus $\kappa$ is taken to be 1,000 , to better model the nearly incompressible behavior. Since the steel tubes are much stiffer than the silicone rubber (the shear modulus of the steel tubes is in the order of $80 \mathrm{GPa}$ ), the steel tubes are considered as rigid bodies and treated with the rigid-body constraint formulation discussed in this paper.

Along the lines of Lefevre et al. [27], and making use of the symmetries in the model, we consider only a quarter of the model with a structured quadrilateral mesh, as shown in Fig. 4 (a). In the mesh, the matrix is discretized by quadratic quadrilateral element with continuously linear pressure approximation (resembles the quadratic Taylor-Hood element in fluid problems), which is shown to be numerically stable (at least for linear problems) $[18,19,28]$, whereas the rigid fibers do not contain any element. For comparison purpose, we also work out the problem with the $\mathrm{CPE} 8 \mathrm{H}$ elements in ABAQUS using a mesh that has the same discretization in the matrix, but also with discretization inside the fibers, as shown in Fig. 4 (b). The statistics of the two meshes are presented in Table 1. As we can see, the standard FE approach considerably increases the total number of nodes/elements in the mesh because of the substantial gradation in the mesh size needed to accommodate the different length scales in the model, i.e., the length scale of the defects, fibers and the block. With the rigid-body constraint formulation, 
on the contrary, the total number of elements/nodes in the mesh is significantly reduced because each rigid fiber is described only by the set of constraints of the nodes on its boundary, which greatly improves the computational efficiency. As a result, while the FE system of the standard FE approach has a total of 113,751 DOFs, that of the rigid-body constraint formulation contains only a total of 66,434 DOFs, 639 of which are the Lagrange multipliers associated with the rigid-body constraints ${ }^{1}$. We note that most of the (636 of them) additional Lagrange multipliers are associated with linear constraints and thus can be readily condensed out of the FE system to further improve computational efficiency. Moreover, since all the constraining equations are algebraic, the rigid-body formulation is able to handle the strong gradation in the edge lengths of the fibers, which is a serious problem with the "n-gon" approach because of the quality of the shape functions and numerical integration.

Figure 5 shows the snapshots of the deformed configurations of the region between the fibers obtained by the quadratic quadrilateral elements with continuously linear pressure field at four stages of macroscopic strains, $\bar{\varepsilon}=4.5,12.1,16.5$, and $35.1 \%$. The results obtained by the CPE8H element with ABAQUS is identical and therefore not shown here. As quantitative comparison, we also work out the current-to-undeformed area ratios $a^{i} / A^{i}$ and $a^{m} / A^{m}$ of the defects at the interfaces and midpoints, which are shown in Figs. 6 (a) and (b). The comparison shows good agreement between the results obtained by the rigid-body constraint approach and ABAQUS. The rigid-body constraint approach is able to fully capture the growth history and interactions between the defects, indicating that all the defects start to grow initially; however, further loading results in the contraction of the interfacial defects and the continuing growth of the midpoint defect.

\subsection{Elastomers reinforced with spherical particles}

The second example presents the application of the rigid-body formulation to the study of the nonlinear elas- tic response of a compressible elastomer filled with an isotropic distribution of rigid spherical particles. To that end, we consider a unit cell generated via a random sequential adsorption algorithm [29,3] that contains a total of 30 spherical particles of same radius, resulting in a total volume fraction of roughly $c=20 \%$, as shown in Fig. 7 (a). The unit cell is subjected to uniaxial tension, whose macroscopic deformation has the form

$$
<\mathbf{F}>=\lambda_{1} \mathbf{e}_{1} \otimes \mathbf{e}_{1}+\lambda_{2} \mathbf{e}_{2} \otimes \mathbf{e}_{2}+\lambda_{3} \mathbf{e}_{3} \otimes \mathbf{e}_{3}
$$

with $\lambda_{k}, k=1,2,3$ being the stretch in $\mathbf{e}_{k}$ direction. If the unit cell is isotropic, we have $\lambda_{2}=\lambda_{3}$. In addition, periodic displacement boundary condition is prescribed [3], that is, for $k=1,2,3$,

$$
\begin{aligned}
& u_{k}\left(1, X_{2}, X_{3}\right)-u_{k}\left(0, X_{2}, X_{3}\right)=\langle\mathbf{F}\rangle_{k 1}-\delta_{k 1} \\
& u_{k}\left(X_{1}, 1, X_{3}\right)-u_{k}\left(X_{1}, 0, X_{3}\right)=\langle\mathbf{F}\rangle_{k 2}-\delta_{k 2} \\
& u_{k}\left(X_{1}, X_{2}, 1\right)-u_{k}\left(X_{1}, X_{2}, 0\right)=\langle\mathbf{F}\rangle_{k 3}-\delta_{k 3},
\end{aligned}
$$

and the shear and bulk moduli of matrix is taken to be $\mu=\kappa=1$.

The displacement-based formulation is used on a tetrahedral mesh generated by Netgen [30], as shown in Fig. 7 (b). The mesh consists of 23,679 nodes and 110,281 elements. Again, each particle in the FE mesh is described only by its boundary nodes. For comparison, we also use a mesh that has the similar discretization in the matrix, but with each particle being discretized by tetrahedral elements, which is shown in Fig. 7 (c). In the latter case, we assign a high moduli to each particle, namely, $\kappa=\mu=5,000$. A comparison of the statistics of the two meshes is provided in Table 2. As shown from the comparison, because the rigid-body constraint formulation requires absolutely no discretization in the interior of each particle, it reduces the total numbers of nodes and elements in the mesh as compared to the standard FE approach. In contrast, it is also observed that, due to the additional 17,922 Lagrange multipliers associated with the rigid-body constraints, the resulting total number of DOFs in the FE systems for the rigid-body constraint formulation increases as compared to that for

\footnotetext{
${ }^{1}$ We remark that the total numbers of pressure DOFs for both approaches are different. The pressure field in the rigid-body constraint formulation is continuous(in the matrix) while the one used in the ABAQUS is discontinuous.
} 
the standard FE approach. We note that, since the majority $(17,622)$ of the additional Lagrange multipliers are associated with linear constraints, they can be readily condensed out of the FE system for more efficient computational performance.

Figures 8 shows the deformed configurations at $\lambda_{1}=3$ obtained by both meshes. For the results obtained by the mesh with rigid-body constraint formulation, although most of the regions surrounding the particles (soft material) are highly stretched, all the particles remain unstrained, and the deformed configuration is identical to the one obtained by ABAQUS. For quantitative comparison, we also plot in Figs 9 (a)-(b) the total elastic energy density $\langle W\rangle$ and the component of the macroscopic first Piola-Kirchoff stress $<\mathbf{P}>_{11}$ as a function of the applied stretch $\lambda_{1}$. We note that, in case of the rigidbody formulation, the macroscopic stress $<\mathbf{P}>_{11}$ is computed from the FE solution of stress in the matrix phase in conjunction with the set of Lagrange multipliers $\boldsymbol{\Lambda}^{i}$ and relations (8). Both approaches produce identical macroscopic responses, indicating the effectiveness of the rigid-body constraint formulation in dealing with complex spatial distributions of rigid domains in 3D.

\section{Concluding remarks}

This work extends the FE implementation of a recently proposed rigid-body constraint formulation to $3 \mathrm{D}$ problems [6] considering both displacement-based and mixed variational formulations. By introducing a set of four "reference nodes", any rigid body in 3D that is described by its boundary nodes is modeled as a set of algebraic equations. The stress quantities associated with the rigid body, such as the boundary tractions and the average first Piola-Kirchhoff stress, are then extracted from the Lagrange multipliers of the constraining equations. Two numerical examples are presented, demonstrating that rigid-body constraint formulation is applicable to finite elements of arbitrary geometry and order in 2D and $3 \mathrm{D}$, which may reduce the size of the discretization (therefore the computational cost) and provide improved accuracy.

\section{Acknowledgement}

We acknowledge the support from the US National Science Foundation (NSF) through Grant CMMI \#1624232 (formerly \#1437535). The information presented in this paper is the sole opinion of the authors and does not necessarily reflect the views of the sponsoring agency.

\section{Appendix A: Variational formulation with rigid- body constraints considering body forces}

This appendix presents a possible generalization of the variational formulation with rigid-body constraint including the presence of body force in both matrix and rigid spatial domains (e.g., rigid inclusions). Only the generalization of displacement-based variational formulation is shown here, as that of the mixed formulation follows the same concept and procedures.

If the elastic solid $\Omega$ is assumed to be subjected to a body force $\mathbf{f}$ (per unit undeformed volume), not only the displacement field on the boundary of the rigid domains, $\Gamma^{i}$, but that in the interior of each rigid domain $\Omega_{i}^{(2)}$, needs to be considered. Since the displacement field in the interior of each rigid domain takes the form of rigidbody motion, we consider a slightly modified definition of the restricted set for kinematically admissible displacements $\mathcal{K}^{R}$ :

$$
\begin{array}{r}
\mathcal{K}^{R}=\left\{\mathbf{u}: \text { on } \partial \Omega^{\mathbf{X}}, \mathbf{u}=\mathbf{u}^{0}, \text { and in } \Omega_{i}^{(2)}, i=1, \ldots, N\right. \\
\mathbf{u}=\mathbf{u}_{0}^{i}+\mathbf{H}^{i} \mathbf{X}, \mathbf{H}^{i T} \mathbf{H}^{i}+\mathbf{H}^{i}+\mathbf{H}^{i T}=0 \\
\text { where } \left.\mathbf{u}_{0}^{i} \in \mathbb{R}^{d}, \mathbf{H}^{i} \in \mathbb{R}^{d \times d}\right\}
\end{array}
$$

As a result, the displacement variational principle in (4) and (5) becomes one where we seek the equilibrium displacement field $\widehat{\mathbf{u}} \in \mathcal{K}^{R}$ such that,

$$
\Pi(\widehat{\mathbf{u}})=\min _{\mathbf{u} \in \mathcal{K}^{R}}\left[\Pi^{(1)}(\mathbf{u})-\sum_{i=1}^{N} \int_{\Omega_{i}^{(2)}} \mathbf{f} \cdot\left(\mathbf{u}_{0}^{i}+\mathbf{H}^{i} \mathbf{X}\right) \mathrm{d} \mathbf{X}\right],
$$

where

$$
\begin{array}{r}
\Pi^{(1)}(\mathbf{u})=\int_{\Omega^{(1)}} W^{(1)}(\mathbf{X}, \mathbf{F}(\mathbf{u})) \mathrm{d} \mathbf{X}-\int_{\partial \Omega^{\boldsymbol{\sigma}}} \mathbf{t} \cdot \mathbf{u} \mathrm{d} S \\
-\int_{\Omega^{(1)}} \mathbf{f} \cdot \mathbf{u} \mathrm{d} \mathbf{X}
\end{array}
$$


is the potential energy of phase $\Omega^{(1)}$. Similarly, the Lagrangian of the displacement-based variational formulation in (6) is therefore amended as:

$$
\begin{array}{r}
\min _{\mathbf{u} \in \mathcal{K}\left(\Omega^{(1)}\right)} \min _{\mathbf{u}_{0}^{i} \in \mathbb{R}^{d}} \min _{\mathbf{H}^{i} \in \mathbb{R}^{d \times d}} \operatorname{stat}_{\boldsymbol{\lambda}^{i} \in\left[C^{0}\left(\Gamma^{i}\right)\right]^{d}} \operatorname{stat}_{\boldsymbol{\Sigma}^{i} \in \mathbb{R}_{s y m m}^{d \times d}} \\
\mathcal{L}\left(\mathbf{u}, \mathbf{u}_{0}^{i}, \mathbf{H}^{i}, \boldsymbol{\lambda}^{i}, \boldsymbol{\Sigma}^{i}\right)
\end{array}
$$

with

$$
\begin{aligned}
\mathcal{L}\left(\mathbf{u}, \mathbf{u}_{0}^{i}, \mathbf{H}^{i}, \boldsymbol{\lambda}^{i}, \boldsymbol{\Sigma}^{i}\right) & =\Pi^{(1)}(\mathbf{u}) \\
+\sum_{i=1}^{N} \frac{1}{2}\left|\Omega_{i}^{(2)}\right| \boldsymbol{\Sigma}^{i}:\left(\mathbf{H}^{i T} \mathbf{H}^{i}+\mathbf{H}^{i}+\mathbf{H}^{i T}\right)+ & \sum_{i=1}^{N} \int_{\Gamma^{i}} \boldsymbol{\lambda}^{i} \cdot\left(\mathbf{u}-\mathbf{u}_{0}^{i}-\mathbf{H}^{i} \mathbf{X}\right) \mathrm{d} S \\
& -\sum_{i=1}^{N} \int_{\Omega_{i}^{(2)}} \mathbf{f} \cdot\left(\mathbf{u}_{0}^{i}+\mathbf{H}^{i} \mathbf{X}\right) \mathrm{d} \mathbf{X}
\end{aligned}
$$

It is worthwhile looking at the strong form of the Euler-Lagrange equations of (41), which is given by:

$$
\begin{aligned}
& \operatorname{Div} \mathbf{P}(\mathbf{X})=\mathbf{0} \text { in } \Omega^{(1)}, \\
& \mathbf{P}(\mathbf{X}) \mathbf{N}=\mathbf{t} \text { on } \partial \Omega^{\sigma}, \\
& \boldsymbol{\lambda}^{i}-\mathbf{P}(\mathbf{X}) \mathbf{N}^{\Gamma}=\mathbf{0} \text { on } \Gamma^{i}, i=1, \ldots, N, \\
& \int_{\Gamma_{i}} \lambda^{i} \mathrm{~d} S-\int_{\Omega_{i}^{(2)}} \mathbf{f d} \mathbf{X}=\mathbf{0}, i=1, \ldots, N, \\
& \left(\mathbf{H}^{i}+\mathbf{I}\right) \boldsymbol{\Sigma}^{i}=\frac{1}{\left|\Omega_{i}^{(2)}\right|}\left[\int_{\Gamma^{i}}\left(\boldsymbol{\lambda}^{i} \otimes \mathbf{X}\right) \mathrm{d} S\right. \\
& \left.\quad-\int_{\Omega_{i}^{(2)}}(\mathbf{f} \otimes \mathbf{X}) \mathrm{d} \mathbf{X}\right], i=1, \ldots, N, \\
& \mathbf{u}=\mathbf{u}_{0}^{i}+\mathbf{H}^{i} \mathbf{X} \text { on } \Gamma^{i}, i=1, \ldots, N, \\
& \mathbf{H}^{i T} \mathbf{H}^{i}+\mathbf{H}^{i}+\mathbf{H}^{i T}=\mathbf{0}, i=1, \ldots, N,
\end{aligned}
$$

where $\mathbf{N}$ is the outward unit normal vector on $\partial \Omega^{\boldsymbol{\sigma}}$, and $\mathbf{N}^{\Gamma}$ is the unit normal vector on $\Gamma$ pointing towards the non-rigid domain $\Omega^{(1)}$.

As compared to the original formulation derived in [6] and Section 2 of the present paper, the body force $\mathbf{f}$ shows up in the additional terms in Eqs. (45) and (46). From the Euler-Lagrange equations, we can observe that the Lagrange multiplier $\boldsymbol{\lambda}^{i}$ is still the traction field on the boundary of the interface $\Gamma^{i}$. Moreover, by applying the
Divergence Theorem to Eq. (46), we have for $i=1, \ldots, N$

$$
\begin{aligned}
\left(\mathbf{H}^{i}+\mathbf{I}\right) \boldsymbol{\Sigma}^{i} & \left.=\frac{1}{\left|\Omega_{i}^{(2)}\right|}\left[\int_{\Gamma^{i}} \mathbf{P N} \otimes \mathbf{X} \mathrm{d} S-\int_{\Omega_{i}^{(2)}} \mathbf{f} \otimes \mathbf{X}\right) \mathrm{d} \mathbf{X}\right] \\
& =\frac{1}{\left|\Omega_{i}^{(2)}\right|} \int_{\Omega_{i}^{(2)}}(\operatorname{Div} \mathbf{P} \otimes \mathbf{X}+\mathbf{P}-\mathbf{f} \otimes \mathbf{X}) \mathrm{d} \mathbf{X} \\
& =\frac{1}{\left|\Omega_{i}^{(2)}\right|} \int_{\Omega_{i}^{(2)}} \mathbf{P} \mathrm{d} \mathbf{X},
\end{aligned}
$$

suggesting that the Lagrange Multiplier $\boldsymbol{\Sigma}^{i}$ is also the volume average of the Second Piola-Kirchhoff stress over the rigid domain $\Omega_{i}^{(2)}$ as in (8).

As a final remark regarding the finite element implementation of the variational principle considering the presence of body force, we comment that we need to treat the following terms in addition to those discussed in Section 3 :

$$
\begin{aligned}
& \int_{\Omega^{(1)}} \mathbf{f} \cdot \mathbf{u} \mathrm{d} \mathbf{X} \\
& \int_{\Omega_{i}^{(2)}} \mathbf{f} \cdot\left(\mathbf{u}_{0}^{i}+\mathbf{H}^{i} \mathbf{X}\right) \mathrm{d} \mathbf{X} .
\end{aligned}
$$

Because the first term integrates over the matrix phase $\Omega^{(1)}$, it is treated with the standard finite element procedures [19, 21, 22, 23]. One the other hand, we also need to compute the volume integral of $\mathbf{f} \cdot\left(\mathbf{u}_{0}^{i}+\mathbf{H}^{i} \mathbf{X}\right)$ over each rigid domain $\Omega_{i}^{(2)}$, as shown in the second term in (50). In practice, such integral can be readily computed using the approximations proposed in the VEM, see, e.g., [7].

\section{Appendix B: Nomenclature}

$\begin{array}{ll}<\mathbf{F}> & \text { Macroscopic deformation gradient } \\ <\mathbf{P}> & \text { Macroscopic first Piola-Kirchhoff stress } \\ <W> & \text { Macroscopic stored-energy function } \\ \Lambda^{i} & \text { Array of nodal tractions for } \Omega_{i h}^{(2)} \\ \lambda^{i} & \text { Lagrange multiplier introduced which is } \\ & \text { a continuous vector field on } \Gamma^{i} \\ \boldsymbol{\Sigma}^{i} & \text { Lagrange multiplier introduced for } \Gamma^{i} \\ & \text { which is a constant symmetric second } \\ & \text { order tensor } \\ \Gamma^{i} & \text { The interface between the rigid domain } \\ & \Omega_{i}^{(2)} \text { and non-rigid domain } \Omega^{(1)} \\ \Gamma_{h}^{i} & \text { The boundary of the } i \text { th discrete rigid } \\ & \text { domain } \Omega_{i h}^{(2)}\end{array}$


$\lambda_{1}$

$\lambda_{2}$

$\lambda_{3}$

$\mathcal{K}^{R}$

$\mathcal{K}_{h, k}$

$\mathcal{P}_{k}(E)$

$\mathcal{Q}$

$\mathcal{Q}_{h, k-1}$

$\mathcal{V}_{k}(E)$

$\mathcal{W}_{k}\left(\Gamma_{h}^{i}\right)$

$\mu$

$\Omega$

$\Omega^{(1)}$

$\Omega_{h}^{(1)}$

$\Omega_{i}^{(2)}$

$\Omega_{i h}^{(2)}$

$\Omega_{h}$

$\bar{\Pi}(\mathbf{u}, p)$

$\bar{W}(\mathbf{X}, \overline{\mathbf{F}}, J)$

$\bar{W}^{*}(\mathbf{X}, \overline{\mathbf{F}}, p) \quad$ Function obtained by Legendre trans-

$\partial \Omega^{\sigma}$

$\partial \Omega^{\mathbf{X}}$

$\phi_{r}^{i}(\mathbf{X})$

$\Pi(\mathbf{u})$ formation of $J$ in $\bar{W}(\mathbf{X}, \overline{\mathbf{F}}, J)$

Initial bulk modulus

Macroscopic stretch in $\mathbf{e}_{1}$ direction

Macroscopic stretch in $\mathbf{e}_{2}$ direction

Macroscopic stretch in $\mathbf{e}_{3}$ direction

A restricted set of kinematically adimissble displacmenets considering rigid subdomains

Discrete global displacement space of order $k$

Polynomial space of order $k$ on element

E

Space consists of square integrable functions

Discrete global pressure space of order $k-1$

X

$\widehat{\mathbf{u}}$

element $E$

Discrete space for the Lagrange multiplier $\lambda_{h}^{i}$

Initial shear modulus

Undeformed domain

Non-rigid material phase

A discretization of the non rigid domain $\Omega^{(1)}$

The $i$ th constitutively rigid regions of the domain $\Omega$

The $i$ th discrete rigid domain

A discretization of the domain $\Omega$

Potential energy in the two-field mixed formulation

A general stored-energy function such that $W(\mathbf{X}, \mathbf{F})=\bar{W}(\mathbf{X}, \overline{\mathbf{F}}, J)$ when $J=\operatorname{det} \mathbf{F}$

Part of the domain boundary where traction $\mathbf{t}$ is prescribed

Part of the domain boundary where displacement $\mathbf{u}^{0}$ is prescribed

The set of interpolation functions introduced for $\Omega_{i h}^{(2)}$

Potential energy in the displacement-

F

f

$\mathbf{H}^{i}$

$\mathbf{P}$

t

$\mathbf{U}^{i}$

$\mathbf{u}_{0}^{i}$

$\tilde{\mathbf{X}}_{r}^{i}$

Coefficient matrix defined for $\Omega_{i h}^{(2)}$ in forming rigid-body constraints

$\widehat{p}$

$W(\mathbf{X}, \mathbf{F})$

stat

\section{References}

[1] J. Moraleda, J. Segurado, J. LLorca, Finite deformation of incompressible fiber-reinforced elastomers: A computational micromechanics approach, Journal of the Mechanics and Physics of Solids 57 (9) (2009) 1596-1613.

[2] J. Michel, O. Lopez-Pamies, P. Ponte Castañeda, N. Triantafyllidis, Microscopic and macroscopic instabilities in finitely strained fiber-reinforced elastomers, Journal of the Mechanics and Physics of Solids 58 (11) (2010) 1776-1803.

[3] O. Lopez-Pamies, T. Goudarzi, K. Danas, The nonlinear elastic response of suspensions of rigid inclusions in rubber: Ii a simple explicit approximation for finite-concentration suspensions, Journal of the Mechanics and Physics of Solids 61 (2013) 19-37.

[4] T. Goudarzi, D. W. Spring, G. H. Paulino, O. Lopez-Pamies, Filled elastomers: A theory of filler reinforcement based on hydrodynamic and interphasial effects, Journal of the Mechanics and Physics of Solids 80 (2015) 37-27.

[5] H. Chi, C. Talischi, O. Lopez-Pamies, G. H. Paulino, Polygonal finite elements for finite elasticity, International Journal for Numerical Methods in Engineering 101 (2015) 305-328.

[6] H. Chi, O. Lopez-Pamies, G. Paulino, A variational formulation with rigid-body constraints for finite elasticity: Theory, finite element implementation, and applications, Computational Mechanics 57 (2016) 325-338. 
[7] L. Beirão Da Veiga, F. Brezzi, A. Cangiani, G. Manzini, L. D. Marini, A. Russo, Basic principles of virtual element methods., Mathematical Models and Methods in Applied Sciences 23 (1) (2013) 199-214.

[8] L. Beirão Da Veiga, F. Brezzi, L. D. Marini, Virtual Elements for linear elasticity problems., SIAM Journal on Numerical Analysis 51 (2) (2013) 794-812.

[9] A. L. Gain, C. Talischi, G. H. Paulino, On the virtual element method for three-dimensional elasticity problems on arbitrary polyhedral meshes, Computational Methods in Applied Mechanics and Engineering 282 (2014) 132-160.

[10] C. Talischi, G. H. Paulino, Addressing integration error for polygonal finite elements through polynomial projections: A patch test connection, Mathematical Models and Methods in Applied Sciences 24 (8) (2014) 1701-1727.

[11] L. Beirão da Veiga, C. Lovadina, D. Mora, A virtual element method for elastic and inelastic problems on polytope meshes, Computational Methods in Applied Mechanics and Engineering 295 (1) (2015) 327-346.

[12] D. P. Bertsekas, Nonlinear programming, Athena scientific, 1999.

[13] J. C. Simo, R. L. Taylor, K. S. Pister, Variational and projection methods for the volume constraint in finite deformation elasto-plasticity, Computer Methods in Applied Mechanics and Engineering 51 (1) (1985) 177-208.

[14] T. Sussman, K. J. Bathe, A finite element formulation for nonlinear incompressible elastic and inelastic analysis, Computers \& Structures 26 (1) (1987) 357-409.

[15] U. Brink, E. Stein, On some mixed finite element methods for incompressible and nearly incompressible finite elasticity, Computational Mechanics 19 (1) (1996) 105-119.

[16] T. Y. P. Chang, A. F. Saleeb, G. Li, Large strain analysis of rubber-like materials based on a perturbed Lagrangian variational principle, Computational mechanics 8 (4) (1991) 221233.

[17] J. S. Chen, W. Han, C. T. Wu, W. Duan, On the perturbed
Lagrangian formulation for nearly incompressible and incompressible hyperelasticity, Computer Methods in Applied Mechanics and Engineering 142 (3) (1997) 335-351.

[18] D. Boffi, F. Brezzi, M. Fortin, Mixed finite element methods and applications, Springer, 2013.

[19] T. J. R. Hughes, The finite element method: linear static and dynamic finite element analysis, Courier Dover Publications, 2012.

[20] C. Taylor, P. Hood, A numerical solution of the Navier-Stokes equations using the finite element technique, Computers \& Fluids 1 (1) (1973) 73-100.

[21] P. Wriggers, Nonlinear finite element methods, Vol. 4, Springer, 2008.

[22] J. Bonet, R. D. Wood, Nonlinear continuum mechanics for finite element analysis, Cambridge Univ Press, 2008.

[23] O. C. Zienkiewicz, R. L. Taylor, The finite element method for solid and structural mechanics, Butterworth-Heinemann, 2005.

[24] R. D. Cook, D. S. Malkus, M. E. Plesha, R. J. Witt, Concepts and Applications of Finite Element Analysis, 4th Edition, John Wiley \& Sons, Ltd., 2002.

[25] A. Gent, Cavitation in rubber: A cautionary tale, Rubber Chemistry and Technology 63 (3) (1990) 49-53.

[26] K. Cho, A. N. Gent, Cavitation in model elastomeric composites, Journal of materials science 23 (1) (1988) 141-144.

[27] V. Lefevre, K. Ravi-Chandar, O. Lopez-Pamies, Cavitation in rubber: An elastic instability or a fracture phenomenon?, International Journal of Fracture (2014) 1-23.

[28] D. Chapelle, K. J. Bathe, The inf-sup test, Computers \& structures 47 (4) (1993) 537-545.

[29] J. Segurado, J. Llorca, A numerical approximation to the elastic properties of sphere-reinforced composites, Journal of the Mechanics and Physics of Solids 50 (10) (2002) 2107-2121.

[30] J. Schöberl, NETGEN An advancing front 2D/3D-mesh generator based on abstract rules, Computing and Visualization in Science 1 (1) (1997) 41-52. 


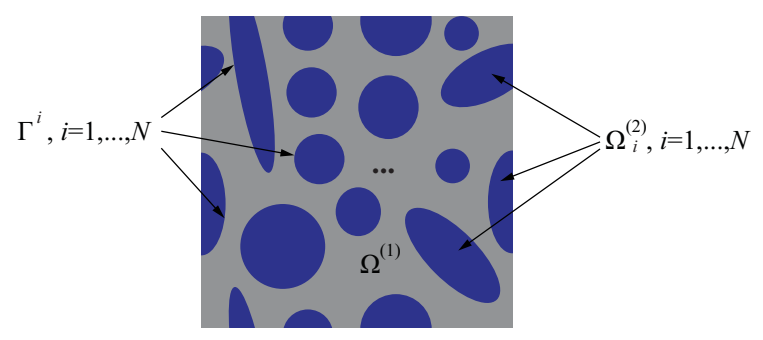

Figure 1: Schematic of an elastic solid containing $N$ disconnected regions $\Omega_{1}^{(2)}, \ldots, \Omega_{N}^{(2)}$ that are constitutively rigid. The interface of material discontinuity between the rigid domain $\Omega_{i}^{(2)}$ and the non-rigid part of the solid $\Omega^{(1)}$ is denoted by $\Gamma^{i}$.

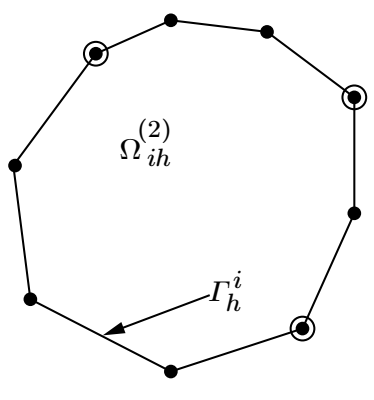

(a)

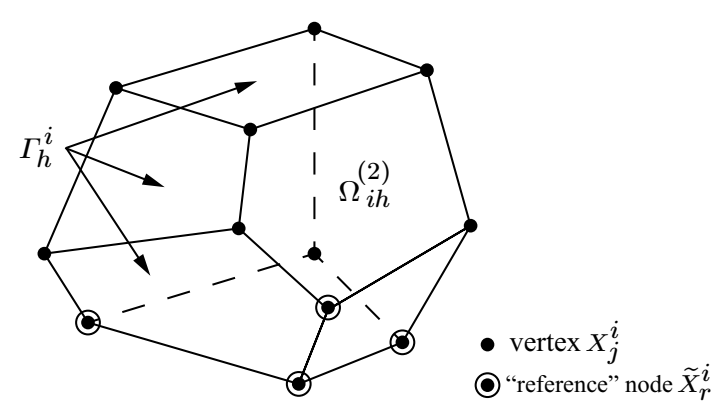

(b)

Figure 2: Illustration of the particles considered by the rigid-body constraint formulations in (a) 2D and (b) 3D. The "reference nodes" are marked with circles. 


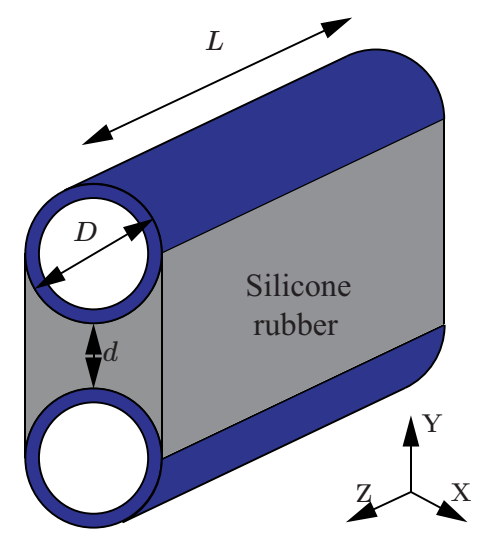

(a)

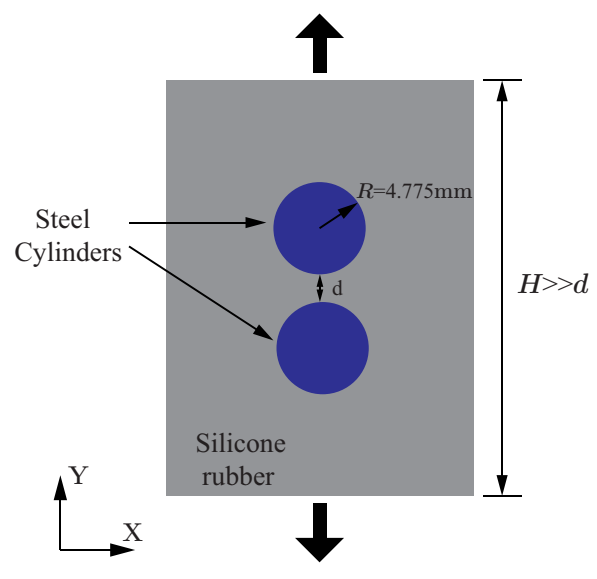

(c)

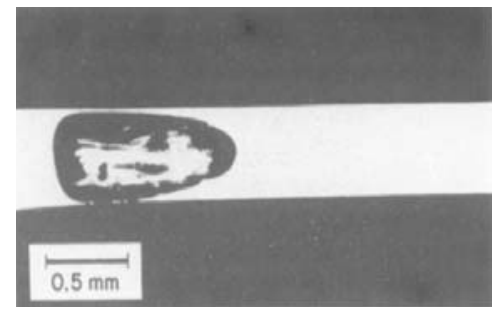

(b)

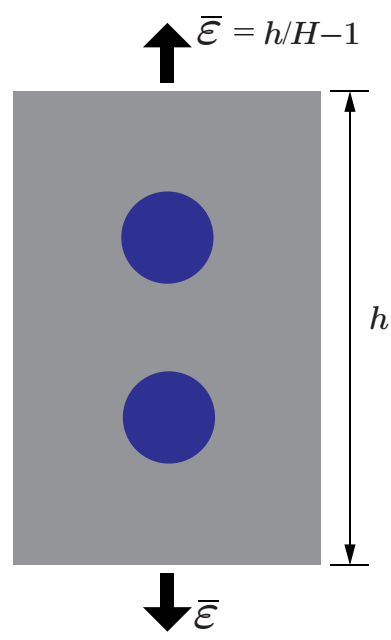

(d)

Figure 3: (a) Schematic of the experimental setup of Cho and Gent [26] with $L=12.5 \mathrm{~mm}, D=9.5 \mathrm{~mm}$ and $d=0.43 \mathrm{~mm}$; (b) A photograph of the lateral view (X direction) of the cavity formed between the two steel tubes for the case of $d=0.43 \mathrm{~mm}$ [26]; (c) A two-dimensional plane-strain model of the experiment in the undeformed configuration; (d) A two-dimensional plane-strain model of the experiment in the deformed configuration. 

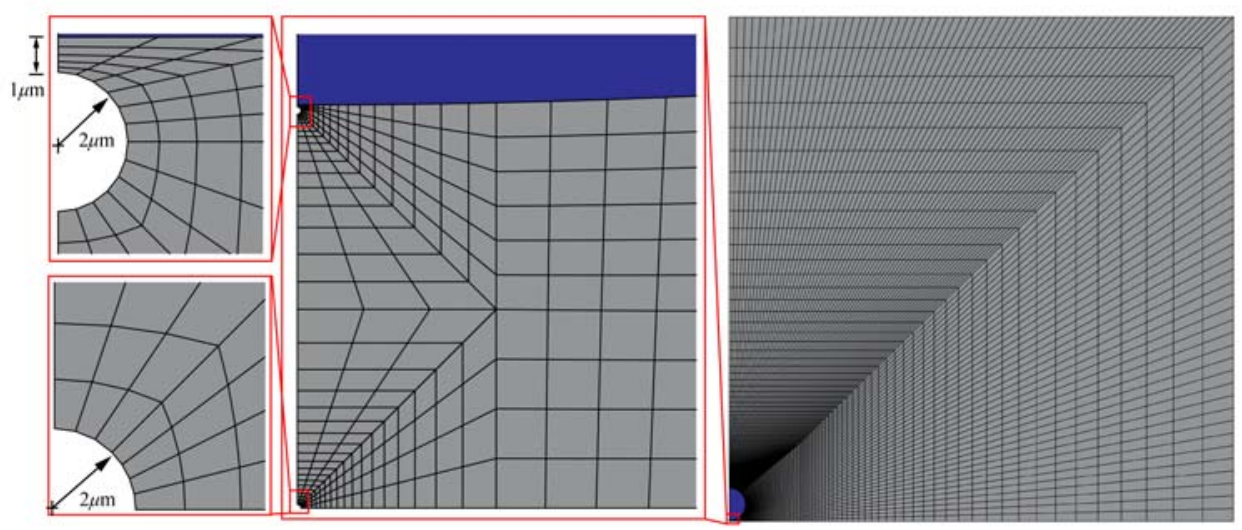

(a)

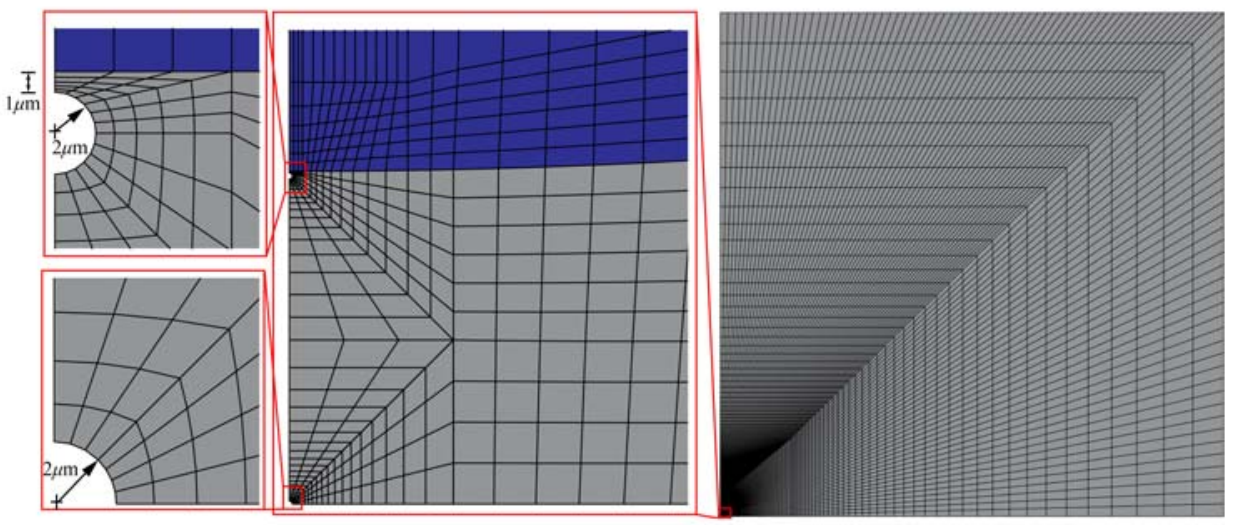

(b)

Figure 4: (a) The 2D structured mesh used with the rigid-body constraint formulation, which consists of 9,201 elements and 28,158 nodes note that interior of the fiber does not contain any elements; (b) The structured mesh used in ABAQUS containing 16111 elements and 48820 nodes. The mesh is the same as (a) in the matrix, but contains additional elements and nodes in the fiber.
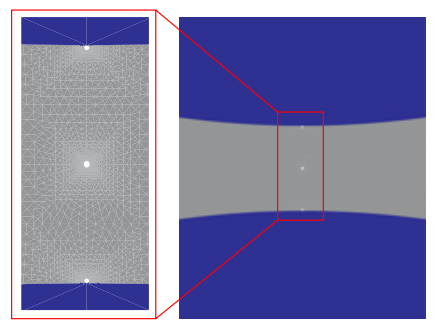

$\bar{\varepsilon}=4.5 \%$

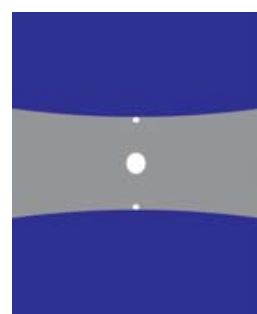

$\bar{\varepsilon}=12.1 \%$

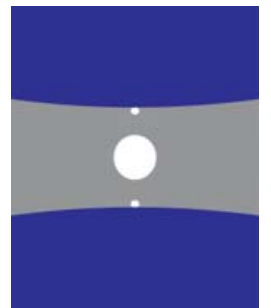

$\bar{\varepsilon}=16.5 \%$

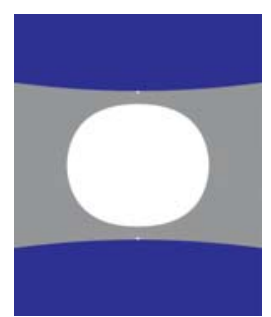

$\bar{\varepsilon}=35.1 \%$

Figure 5: With three defects introduced in the model, the deformed configurations of the region between two fibers under four levels of macroscopic strains. 


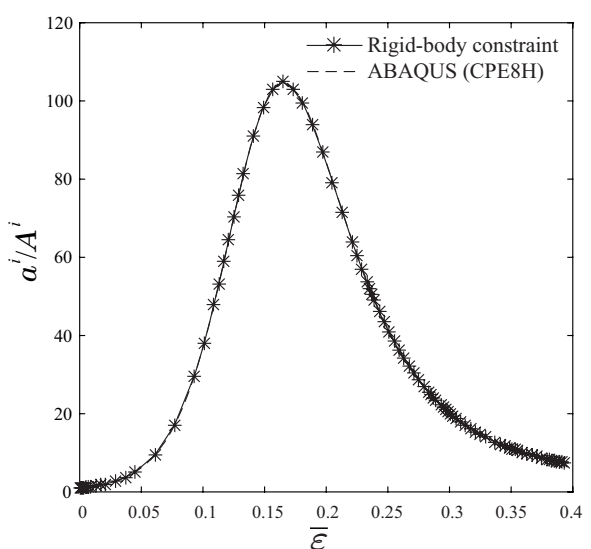

(a)

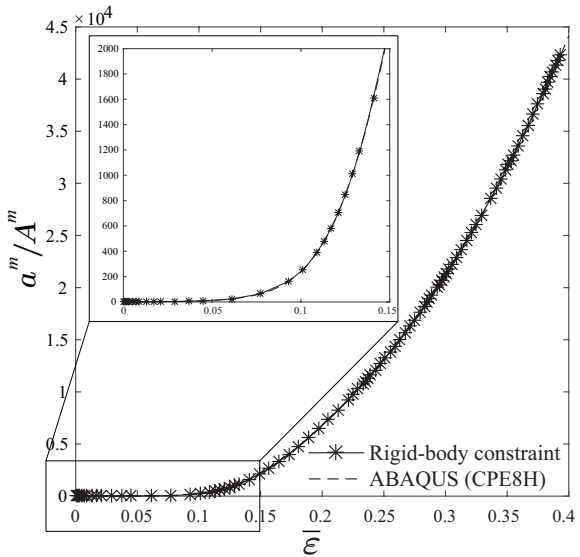

(b)

Figure 6: The growth histories of the three defects quantified by the ratio of their deformed areas to their initial areas as function of the applied macroscopic strain $\bar{\varepsilon}$ : (a) the defects on the matrix/fiber interface, and (b) the defect in the middle of the two fibers. 


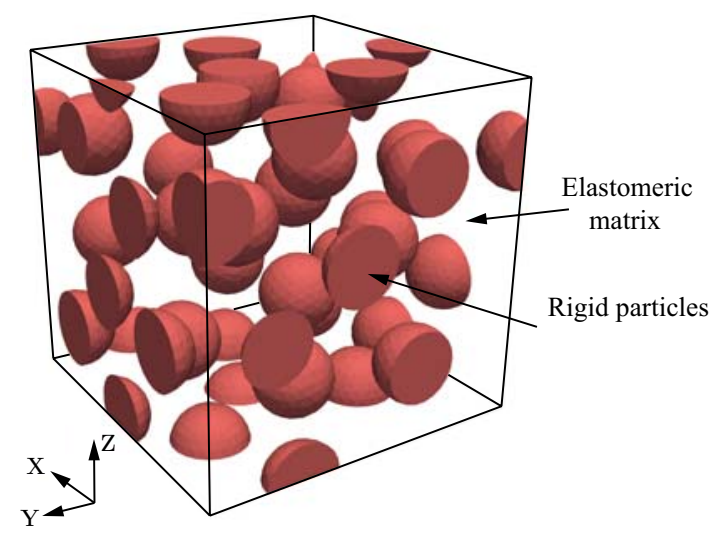

(a)

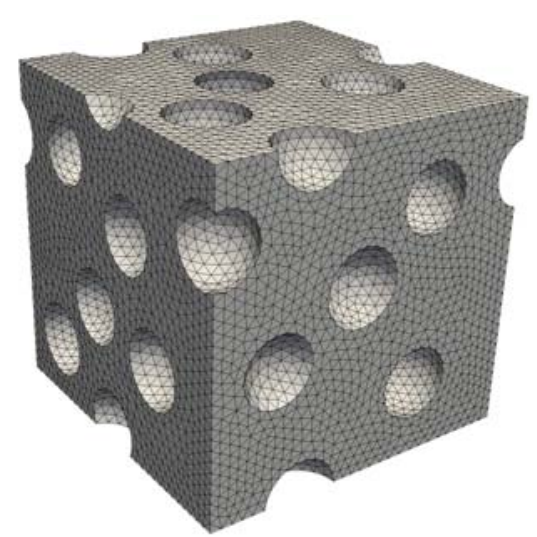

(b)

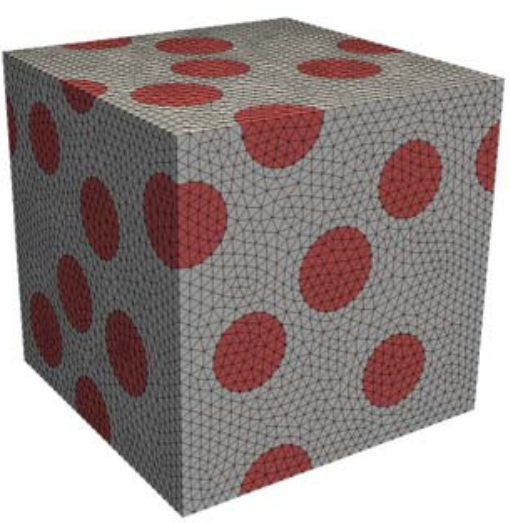

(c)

Figure 7: (a) The unit cell considered in this example, which contains 30 monodispersed spherical particles at a total volume fraction $c=20 \%$. (b) The mesh considered with the rigid-body constraint formulation, which consists of 110281 elements and 23679 nodes (note that none of the inclusions is discretized). (c) The mesh used in ABAQUS that contains 131,551 elements and 25,515 nodes (note that all the inclusions are discretized). 


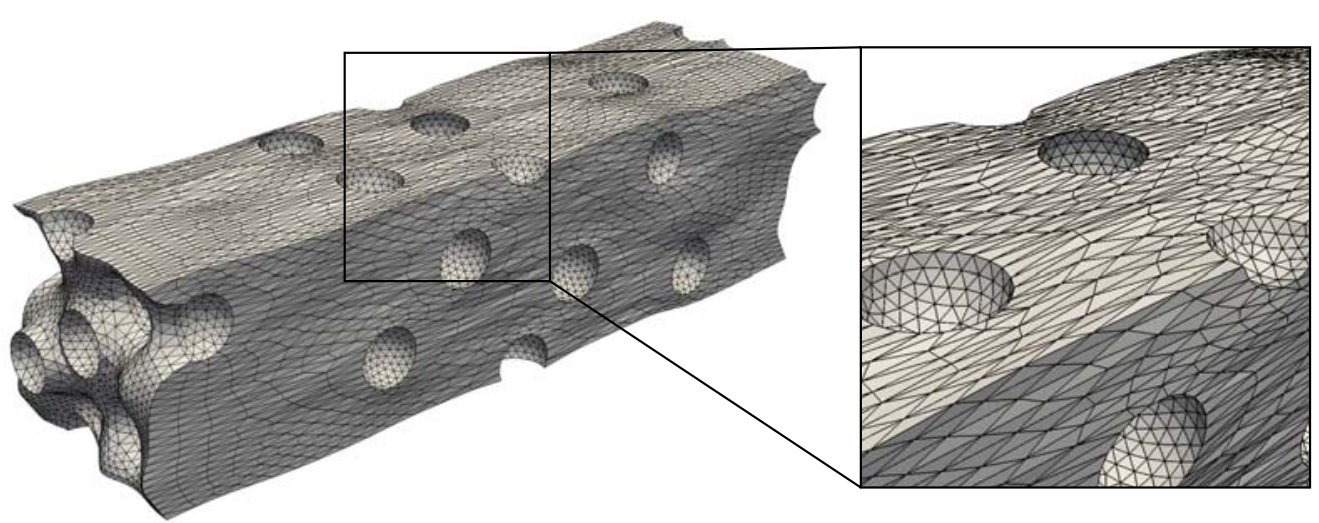

(a)

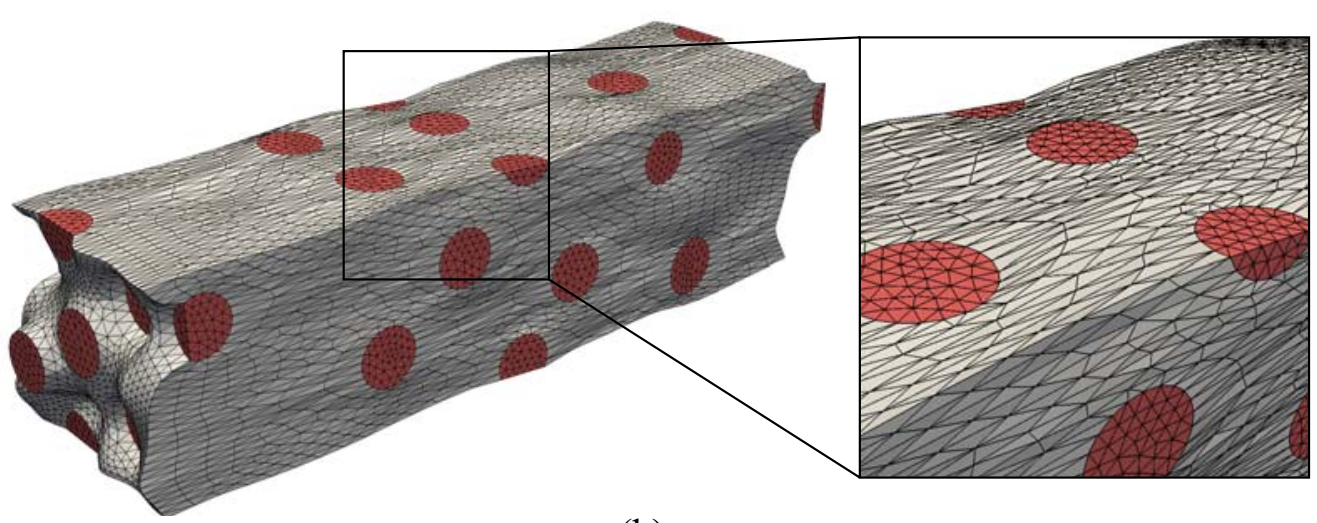

(b)

Figure 8: Deformed shape of the unit cell at stretch $\lambda_{1}=3$ obtained by (a) rigid-body constraint formulation (no discretization of inclusions needed) and (b) ABAQUS (discretization of inclusions is required).

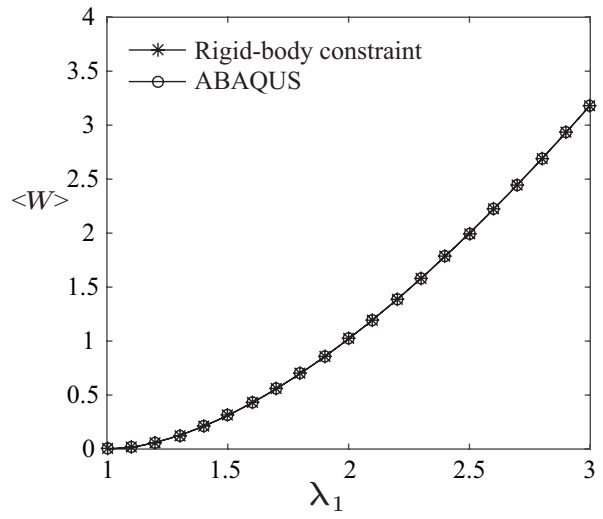

(a)

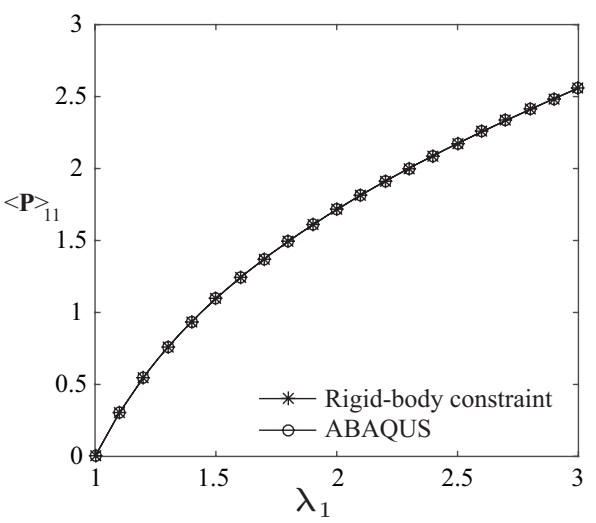

(b)

Figure 9: Macroscopic quantities as function of applied stretch $\lambda_{1}$ : (a) macroscopic stored-energy function and (b) macroscopic first Piola-Kirchhoff stress. 
Table 1: The statistics of meshes in Example 1

\begin{tabular}{|c|c|c|c|c|}
\hline Approaches & \# of Nodes & \# of Elem. (Matrix) & \# of Elem. (Particles) & Total \# of DOFs \\
\hline Rigid-body constraint & 28,158 & 9,201 & 0 & 66,434 \\
Standard FE approach & 48,820 & 9,201 & 6,910 & 113,751 \\
\hline
\end{tabular}

Table 2: The statistics of meshes in Example 2

\begin{tabular}{|c|c|c|c|c|}
\hline Approaches & \# of Nodes & \# of Elem. (Matrix) & \# of Elem. (Particles) & Total \# of DOFs \\
\hline Rigid-body constraint & 23,679 & 110,281 & 0 & 95,559 \\
Standard FE approach & 25,515 & 109,551 & 22,000 & 85,995 \\
\hline
\end{tabular}

